Der folgende Artikel von Helsana resümiert die zentralen Erkenntnisse ihres neuen Reports «Ausgabenentwicklung in der Gesundheitsversorgung 2016». Der Beitrag beleuchtet die Kostenentwicklung in der Obligatorischen Krankenpflegeversicherung basierend auf den Leistungsdaten von Helsana.

\title{
Ausgabenentwicklungen in der Schweizer Gesundheitsversorgung
}

\section{Mathias Früh}

Politik und Ökonomie, Helsana-Gruppe

Die Schweiz hat ein qualitativ überzeugendes Gesundheitswesen, beim Zugang sind wir sogar Weltspitze. Doch das hat bekanntlich seinen Preis: Innerhalb von sechs Jahren (2008 bis 2014) stiegen die Gesundheitskosten um 10 Milliarden auf gut 71 Milliarden; die Schweiz belegt hier im weltweiten Vergleich sowohl pro Kopf als auch im Verhältnis zum Bruttoinlandprodukt Spitzenplätze ${ }^{1}$. Doch woher kommen diese Kosten?

1 OECD Health at a Glance: Europe 2016: 115/117 (http://bit.ly/2kOgolO).

2 www.helsana.ch/ ausgabenreport
Krankenversicherer sind - als grösster Kostenträger - an Effizienz und Wirtschaftlichkeit des Systems interessiert. Die Leistungsdaten, zu welchen wir Zugang haben,
Verwendung des monatlichen Bruttoeinkommens im Durchschnittshaushalt

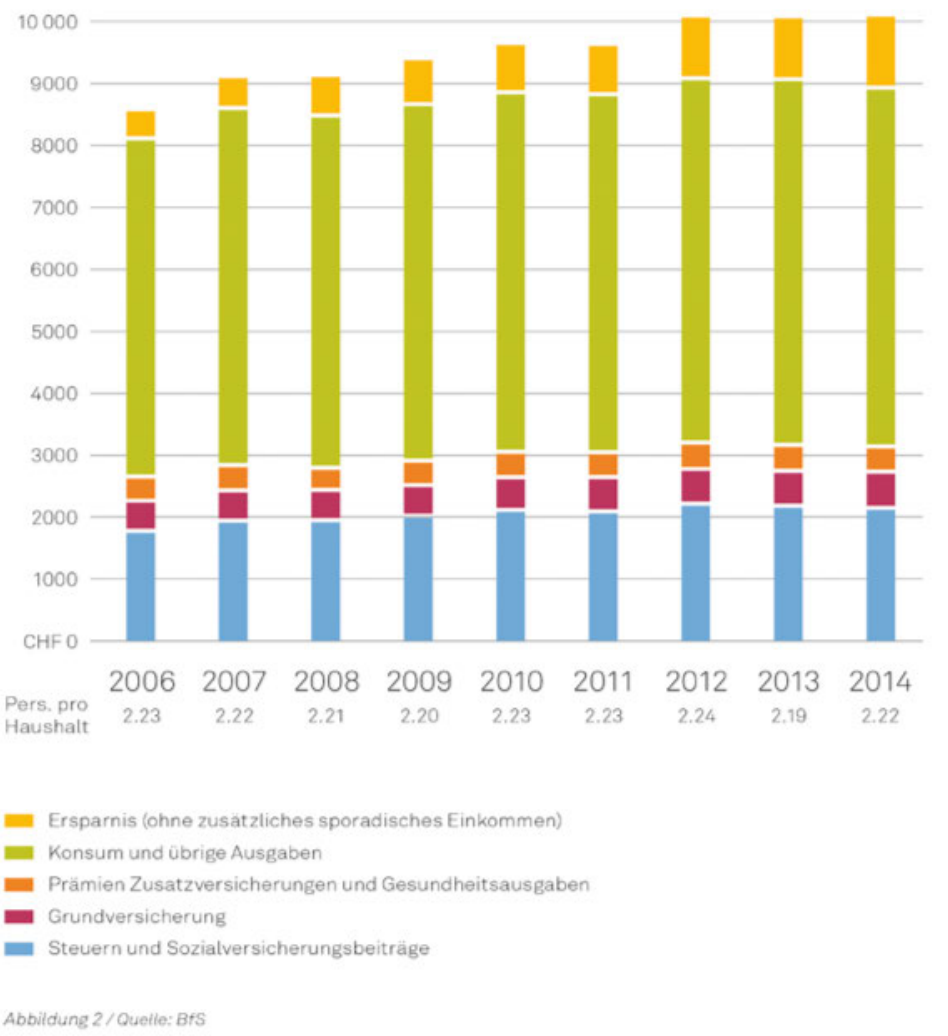

erlauben es, einen vertieften Blick auf die Kostenentwicklung zu werfen und die Ursachen für die Kostenentwicklung zu benennen: Die Demografie - das Bevölkerungswachstum und der wachsende Anteil der älteren Bevölkerung - spielen eine wichtige Rolle bei der Erklärung. Weitere wesentliche Gründe sind das Mengenwachstum und die Ausweitung des Leistungskatalogs sowie Fehlanreize im System, wobei diese schwierig zu quantifizieren sind. Auch die Patienten tragen dazu bei, indem sie häufig- und möglichst sofort - die beste Behandlung verlangen.

Der jüngst erschienene Report «Ausgabenentwicklungen in der Gesundheitsversorgung» ${ }^{2}$ sorgt für zusätzliche Transparenz im Grundversicherungsbereich. Er basiert - wie auch der inzwischen mehrfach erschienene Arzneimittelreport - auf Versicherungsdaten der Obligatorischen Krankenversicherung der HelsanaGruppe und auf öffentlich zugänglichen Statistiken. Die wichtigsten Erkenntnisse werden hier vorgestellt und diskutiert.

\section{Als Volkswirtschaft können wir uns das Gesundheitswesen immer besser leisten}

Die Jahr für Jahr ansteigenden Krankenversicherungsprämien belasten viele Haushalte. Das ist nicht von der Hand zu weisen. Die Belastung des Durchschnittshaushalts durch die Grundversicherung betrug 2014 585 Franken pro Monat, also 7020 Franken im Jahr. Der Anstieg der monatlichen Belastung von 2006 bis 2014 beträgt total rund 100 Franken. Das ist viel und doch wieder nicht, wenn man die Zahlen ins Verhältnis 
setzt: Der monatliche Durchschnittskonsum nahm im selben Zeitraum um 325 Franken zu, die Steuerbelastung sogar um das Vierfache und trotzdem konnten die durchschnittlichen Ersparnisse pro Monat um 715 Franken gesteigert werden (siehe Abb. 1).

Dies ist eine aggregierte Durchschnittsbetrachtung;

Das grosse Problem ist nicht die Tragbarkeit der Kosten als Volkswirtschaft, sondern die Lastenverteilung.

als Volkswirtschaft können wir uns die Kosten des Gesundheitswesens also immer besser leisten. Doch ein Fakt ist auch, dass Durchschnitte für den Einzelnen wenig bedeuten. Denn es gibt immer mehr Haushalte, für welche die Krankenversicherungsprämien eine Höhe erreicht haben, die nicht oder kaum mehr tragbar ist. In diesem Kontext sind auch die permanent aufflammenden Diskussionen in der öffentlichen Agenda zu sehen: Das grosse Problem ist nicht die Tragbarkeit der Kosten als Volkswirtschaft, sondern die Lastenverteilung.

Finanzierung des Gesundheitswesens nach Finanzierungsregimes
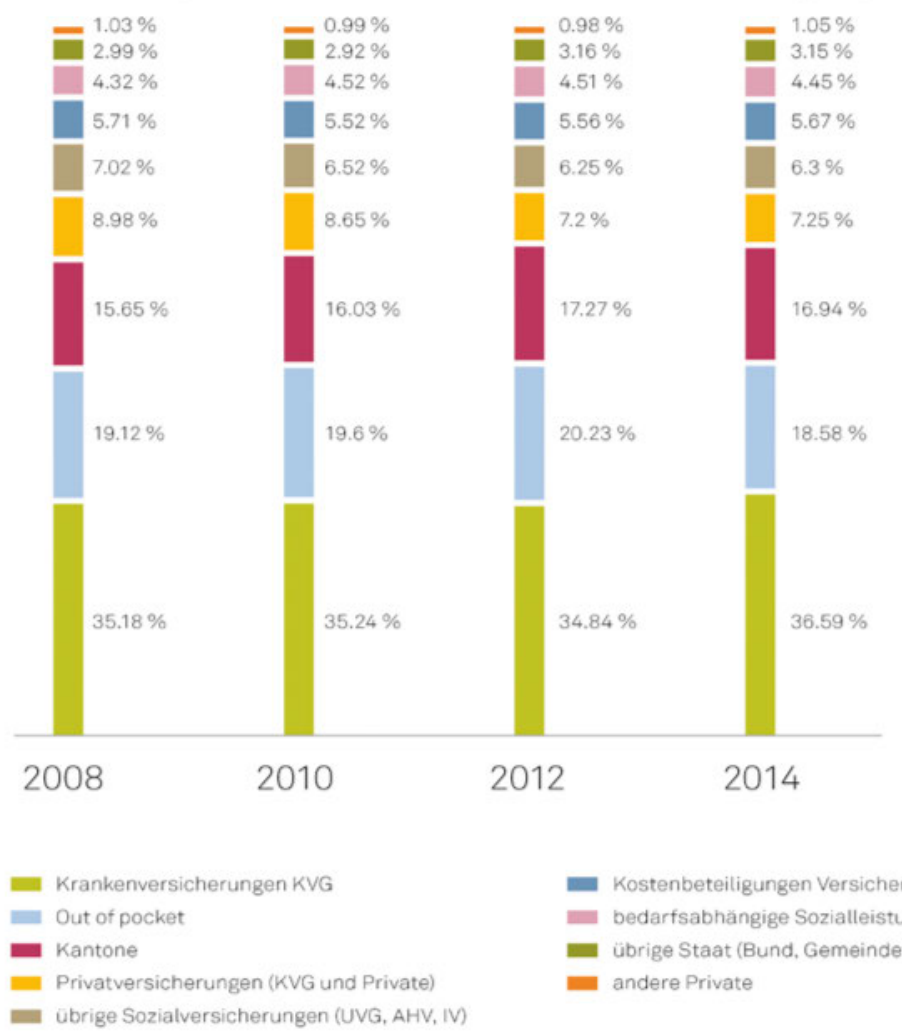

\section{Leistungen zulasten der Grundversiche- rung nehmen stetig $\mathrm{zu}$...}

Dass die Gesundheitskosten zulasten der Allgemeinheit laufend ansteigen, hat auch damit zu tun, dass der Grundversicherungskatalog immer stärker ausgeweitet wird. In der Tendenz verdrängen die solidarischen Finanzierungsformen zunehmend die privaten. Der Finanzierungsanteil der Grundversicherung an den Gesamtkosten des Gesundheitswesens stieg zwischen 2012 und 2014 um rund eine Milliarde Franken (auf 36,6 Prozent der Gesamtkosten, siehe Abb. 2).

\section{... und Patienten werden häufiger und intensiver behandelt}

Der grösste Teil des Wachstums der Versicherungsleistungen von 2008 bis 2015 ist auf die gestiegenen Kosten pro Patient zurückzuführen. Während die Bruttoleistungen um insgesamt 33 Prozent zugenommen haben, sind sie pro Patient um 22 Prozent gestiegen. Das heisst: Zwei Drittel des Leistungskostenwachstums ist auf eine Leistungszunahme pro Patient zurückzuführen. Da diese Zunahme nicht auf Bevölkerungsänderungen basieren, müssen sie bedingt sein durch höhere Tarife, eine Änderung der Behandlungsintensität, Änderungen des Leistungskataloges (z.B. neue, teure Medikamente) und/oder neue Abrechnungsgewohnheiten. Das verbleibende Drittel des OKP-Kostenwachstums ist in einer höheren Patientenzahl begründet, vornehmlich begründet durch das Bevölkerungswachstum.

\section{TARMED schürt intransparente Verrechnungspraxis}

Nun zum TARMED. Der schweizweit wichtigste Tarif im ambulanten Bereich generiert ein stattliches Volumen von über 11 Milliarden Franken. Über 40 Prozent aller TARMED-Leistungen (3,99 Milliarden Taxpunkte) stammen aus dem Kapitel Grundleistungen. Der grösste Teil davon sind Konsultationsleistungen. Rund 15 Prozent der TARMED-Leistungen sind bildgebende Verfahren und weitere 12 Prozent stammen aus der Disziplin Psychiatrie. Die drei grössten Bereiche vereinen demnach um die 7,4 Milliarden auf sich (siehe nachfolgende Tab. 1).

Die Grundleistungen sind von 2012 auf 2015 um 24 Prozent angewachsen. Der Bedarf ist zwar vermutlich bevölkerungsbedingt etwas gestiegen. Allerdings sind die Taxpunkte pro Patient gleichzeitig um 10 Prozent angewachsen. Das deutet darauf hin, dass trotz des Tarifeingriffs des Bundesrates zugunsten der Grund- 


\section{Top 10 Anzahl Taxpunkte pro Kapitel (in Millionen Taxpunkten)}

\begin{tabular}{|c|c|c|c|}
\hline Kapitel & 2012 & 2015 & Änderung seit 2012 \\
\hline Total Taxpunkte & 9'584 & $11 ' 540$ & $+20 \%$ \\
\hline 00-Grundleistungen & 3’987 & 4'943 & $+24 \%$ \\
\hline 39-Bildgebende Verfahren & $1 ' 523$ & 1753 & $+15 \%$ \\
\hline 02-Psychiatrie & 1'182 & $1 ' 403$ & $+19 \%$ \\
\hline 08-Auge & 535 & 683 & $+28 \%$ \\
\hline $\begin{array}{l}\text { 17-Diagnostik und nichtchirurgische } \\
\text { Therapie von Herz und Gefässen }\end{array}$ & 321 & 375 & $+17 \%$ \\
\hline $\begin{array}{l}\text { 35-Operationssaal (OP), } \\
\text { Aufwachraum, Tagesklinik }\end{array}$ & 222 & 270 & $+22 \%$ \\
\hline 32-Radioonkologie, Strahlentherapie & 243 & 270 & $+11 \%$ \\
\hline $\begin{array}{l}\text { 19-Diagnostik und nichtchirurgische } \\
\text { Therapie des Gastrointestinaltraktes }\end{array}$ & 219 & 264 & $+20 \%$ \\
\hline $\begin{array}{l}\text { 37-Klinische Pathologie (Autopsie, } \\
\text { Histologie, Zytologie) und Rechtsmedizin }\end{array}$ & 197 & 237 & $+20 \%$ \\
\hline 04-Haut, Weichteile & 184 & 213 & $+16 \%$ \\
\hline
\end{tabular}

Quelle: Helsana

Tabelle 1: Top 10 Anzahl Taxpunkte pro Kapitel.

versorger im Jahr 2014 von 200 Millionen Franken ein erheblicher Teil der Kostensteigerung einerseits durch neue Verhaltensweisen bei der Verrechnung und anderseits durch ausgedehntere Abklärungen und Behandlungen bedingt ist.

\section{Wir setzen uns dafür ein, dass die überhöhten} Tarife korrigiert werden.

Der TARMED muss - das wissen wir alle - dringend revidiert werden. In seiner heutigen Form benachteiligt er die Grundversorger zugunsten der Spezialisten, lässt eine Beliebigkeit in den Abrechnungen zu und Fehltarifierungen führen zu Fehlanreizen in der Versorgung. Die dringlichen Anpassungen, welche der Bundesrat verordnen will, sind notwendig. Wir setzen uns dafür ein, dass die überhöhten Tarife korrigiert werden, die Transparenz erhöht und ärztlichen Gespräche mit den Patienten aufgewertet werden. Nach einem Tarifeingriff gilt es die völlig veraltete Tarifstruktur der ambulanten medizinischen Versorgung schnell und umfassend zu revidieren und der Realität anzupassen.

\section{Schluss mit reinen Kostendiskussionen: Es braucht Fokus auf Qualität und Effizienz}

Im Gesundheitswesen - wie in unserem Ausgabenreport auch - sind in erster Linie Leistungs- und Kostendaten verfügbar. Auch die derzeitige politische Diskussion dreht sich hauptsächlich um die steigenden Gesundheitskosten. Die bestimmenden Akteure und insbesondere die Gesundheitspolitik reagieren in erster Linie auf die Ausgabenentwicklung, welche als Sündenbock für Verteilungsprobleme herhalten muss. Die Kosten in den Griff zu kriegen ist wichtig, doch notwendig ist zugleich, dass sich das Versorgungssystem und die gestaltende Politik neben den Ausgaben vermehrt an der Verbesserung des Patientennutzens und der Behandlungsqualität orientieren müssen Massnahmen sind also auf Qualitäts- und Effizienzgesichtspunkte auszurichten. Es muss gelingen, Klarheit über das zu bekommen, was am Schluss herauskommt: das Behandlungsergebnis. Nur so lässt sich eine reine Kostendiskussion überwinden, denn das Gesundheitswesen ist weit mehr als nur ein Kostenfaktor. 